\title{
Mechanistic Insights into Immune Suppression and Evasion in Bacterial Vaginosis
}

\author{
Emmanuel Amabebe ${ }^{1}$ (D) Dilly O. C. Anumba ${ }^{1}$ (])
}

Received: 22 August 2021 / Accepted: 18 January 2022 / Published online: 7 February 2022

(c) Crown 2022

\begin{abstract}
The immunological response to bacterial vaginosis (BV) remains poorly understood and recurrent BV is still a major public health burden especially in the pregnant population. This article reviews the potential mechanisms by which BV-associated bacteria suppress and circumvent the host and microbial defence responses, and propagate their survival/dominance without overt inflammation. We discuss the composition of cervicovaginal mucosal barrier and the mechanism by which BV circumvents host defence: the degradation of the mucosal barrier and immunoglobulin A (IgA); the BV-associated organism Gardnerella vaginalis haemolysin (vaginolysin); diminished IgA response against vaginolysin; mucosal sialic acid degradation, foraging and depletion; inhibition of IL-8-induced neutrophilic infiltration; and metabolite-induced incapacitation of neutrophil and monocyte chemotaxis. We also highlight the tolerance/resistance to both host and antimicrobial molecules mounted by BV-associated biofilms. A plausible role of sialic acid-binding immunoglobulin-like lectins (SIGLECS) was also suggested. Sialidase, which is often produced by G. vaginalis, is central to the immunosuppression, relapse and recurrence observed in BV, although it is supported by other hydrolytic enzymes, vaginolysin and immunomodulatory metabolites.
\end{abstract}

\section{Introduction}

Bacterial vaginosis (BV) is a polymicrobial disorder of the lower genital tract characterised by an alteration in the vaginal microenvironment (dysbiosis) resulting in the loss of Lactobacillus species dominance, increase in vaginal $\mathrm{pH}$ and a dramatic overgrowth of pathogenic Gram negative and positive facultative and obligate anaerobic bacteria such as Gardnerella, Atopobium, Bacteroides, Mobiluncus, Prevotella, Mycoplasma, Peptostreptococcus, Anaerococcus, Sneathia, Clostridium, Leptotrichia species, BV-associated bacterium 1 (BVAB1) to BVAB3 etcetera [1-7]. The specific vaginal bacterial composition of $\mathrm{BV}$ can differ between individual women $[5,8,9]$. However, one frequent culprit in the pathogenesis and diagnosis of BV is Gardnerella vaginalis, a non-motile, catalase-negative, Gram variable facultative anaerobic coccobacilli $[6,7]$. Although $G$. vaginalis was

Dilly O. C. Anumba

d.o.c.anumba@sheffield.ac.uk

1 Academic Unit of Reproductive and Developmental Medicine-Obstetrics and Gynaecology, Department of Oncology and Metabolism, The University of Sheffield, 4th Floor, Jessop Wing, Tree Root Walk, Sheffield S10 2SF, UK initially regarded as the sole cause of BV-related clinical signs and symptoms [10], i.e. "primary pathogen model" [7], synergistic contributions from other anaerobic pathogens have been reported more recently [11-13]. Despite its pathogenic potential, G. vaginalis is present in the vagina of most women (including $>60 \%$ without BV), although women with BV have $\sim$ fourfold higher levels compared to women without BV $[14,15]$. Unlike the strict anaerobes such as Prevotella spp., G. vaginalis can adhere to the vaginal epithelium at $\mathrm{pH}$ of $4-5$ and tolerate environments with high redox potential [7]. The BV-associated microbiota has been studied and reported extensively $[1,8,16,17]$ and is beyond the scope of the current report. However, the mechanisms (e.g. sialidase and metabolite activities) employed by G. vaginalis and other anaerobes to contribute to the features and health complications associated with BV [6] are discussed in this review.

The dysbiotic vaginal econiche in BV can be induced by several factors including hormonal changes, menstruation, pregnancy, multiple sex partners, smoking, poor personal hygiene, use of contraceptives, antibiotic therapy, socioeconomic status, psychosocial stress, and some infections and disorders such as diabetes mellitus or insulin resistance [1, $4,18]$. 
$\mathrm{BV}$ is the most common vaginal disorder of reproductiveage women worldwide [3] including premenopausal, fertile and pregnant women [4], with an annual estimated treatment cost of $\$ 4.8$ billion [19]. The economic burden of BV can triple when the cost of BV-associated preterm birth and human immunodeficiency virus (HIV) cases are included [19]. The global prevalence of BV is presented in Table 1[19], and prevalence rates range from 5 to $70 \%[1,17]$. BV is a major public health burden as it is associated with poor reproductive outcomes including preterm birth, low birth weight, chorioamnionitis, amniotic fluid infection, preterm rupture of membranes, miscarriage, failure of in vitro fertilisation, pelvic inflammatory disease, postpartum endometritis and increased risk of acquisition and transmission of HIV and other sexually transmitted infections (STIs) [1, 5, 20-23].

$\mathrm{BV}$ is usually asymptomatic. However, in severe cases symptoms such as vaginal discomfort, and a non-itchy fishy or malodourous homogeneous creamy/greyish vaginal discharge $[1,4,19]$ that may be more noticeable during menstruation or after sexual intercourse have been reported [1]. Many women with BV only complain of malodorous vaginal discharge without an overt inflammation leading to the term "vaginosis" instead of "vaginitis", which is an inflammation of the vagina [24].

$\mathrm{BV}$ is an enigmatic syndrome with controversial aetiology [20]. A decline in the health-promoting Lactobacillus species leads to a decrease in lactic acid that acidifies the vaginal milieu. The increased $\mathrm{pH}$ of the ecosystem creates a conducive environment for the proliferation of mixed

Table 1 Global prevalence of bacterial vaginosis [19]

\begin{tabular}{ll}
\hline Region & $\begin{array}{l}\text { Preva- } \\
\text { lence } \\
(\%)\end{array}$ \\
\hline South Asia & 29 \\
North America & 27 \\
Middle East and North Africa & 25 \\
Sub-Saharan Africa & 25 \\
East Asia and Pacific & 24 \\
Latin America and Caribbean & 24 \\
Europe and Central Asia & 23 \\
\hline
\end{tabular}

There is also considerable racial/ethnic variance in bacterial vaginosis (BV) prevalence. In both general and pregnant population, prevalence of BV is higher in blacks compared with other racial and ethnic groups. Within North America, prevalence of BV in the general population was significantly higher in blacks (33\%) and Hispanic women $(31 \%)$, than white $(23 \%)$ and Asian $(11 \%)$ women [19]. Such disparities may be influenced by socioeconomic factors and biological factors such as lower concentrations of health-promoting vaginal Lactobacillus spp. in black women [35]. The mechanisms underpinning the racial/ethnic differences in the prevalence of BV are crucial and warrants further investigation. However, this is beyond the scope of this review anaerobes that were hitherto kept dormant by lactobacilli and their antimicrobial by-products including lactic acid, $\mathrm{H}_{2} \mathrm{O}_{2}$, bacteriocins and biosurfactants [1]. The resultant heterogeneous vaginal space with $\mathrm{pH}>4.5$, increased bacterial load and species diversity also has increased concentrations of short chain fatty acids - acetate, butyrate, isobutyrate, propionate, formate, succinate; and amines-putrescine, cadaverine, trimethylamine produced by the anaerobes [8, $16,25,26]$. The anaerobes also utilise lactic acid as energy source to further propagate their survival and dominance [8, 25, 27].

Because there is no clear evidence of direct heterosexual transmission of BV-associated bacteria, BV is not usually described as a STI. Instead, due to its positive relationship with frequent unprotected sexual intercourse with new and multiple sexual partners, it has been termed a sexually enhanced infection [24, 27]. However, the reduction in the incidence of $\mathrm{BV}$ with the use of condom, the detection of $\mathrm{BV}$-associated bacteria in the male genital microbiota [28] and relapse after treatment if the woman continues to have unprotected sexual intercourse with the same sex partner are supportive of BV being sexually transmitted [27]. More recently, the spread of dispersed cells or cell aggregates from biofilms between hosts have boosted the putative STI profile of BV [29-33].

BV is diagnosed clinically by the Amsel's criteria, and microbiologically by the Nugent scoring system [1, 27]. Both strategies do not detect polymorphonuclear leukocytes [34] in vaginal fluid due to the absence of an obvious inflammatory response [27]. The absence of an overt inflammatory response has been attributed to the immunomodulatory actions of some dysbiosis-associated metabolites such as acetate and succinate [25]. There is also a coevolutiondetermined immune tolerance between the gut-derived normal and abnormal vaginal microbiota [28].

The lack of clear signs of inflammation (immune response) against the pathogenic bacteria has encouraged the description of BV as a microbiological and immunological conundrum. Hence, we conceptualised this review to examine the plausible mechanisms undergirding immune suppression and evasion in bacterial vaginosis. The composition of cervicovaginal mucosal barrier; degradation of the mucosal barrier and immunoglobulin $\mathrm{A}(\operatorname{Ig} \mathrm{A})$; G. vaginalis haemolysin (vaginolysin); diminished IgA response against vaginolysin; mucosal sialic acid degradation, foraging and overall depletion; inhibition of IL-8-induced neutrophilic infiltration and metabolite-induced incapacitation of neutrophil and monocyte chemotaxis were discussed. We also considered the tolerance/resistance to both host and microbial antimicrobial molecules mounted by BV-associated biofilms. A plausible role of sialic acid-binding immunoglobulin-like lectins (SIGLECS) and therapeutic value of sialidase inhibition were also accentuated. 
Fig. 1 Biochemical composition of mucus and the structure of mucin glycoprotein showing the core, backbone and terminal domains. Mucins are highly glycosylated with carbohydrates making up $85 \%$ of their dry weight and protecting them from proteolysis [37]. FA, fatty acid; Gal, galactose; GalNAc, $N$-acetylgalactosamine; GlcNAc, $\mathrm{N}$-acetylglucosamine; $\operatorname{IgA}$, immunoglobulin A; Neu5Ac, $\mathrm{N}$-acetylneuraminic acid (sialic acid); PL, phospholipid; Pro, proline; Ser, serine; $T h r$, threonine

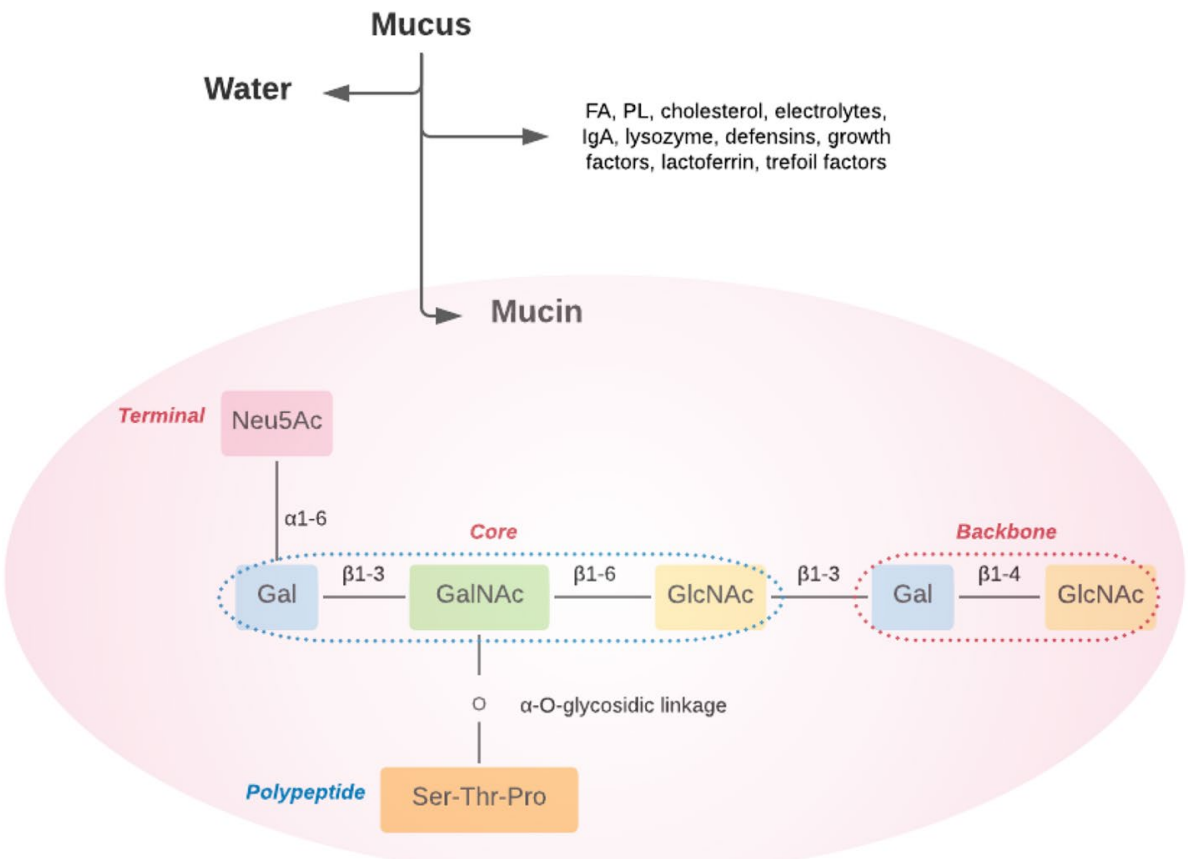

We evaluated the hypothesis that sialidase, supported by immunomodulatory metabolites all produced by BV-associated bacteria, is central to the immunosuppression observed in BV that accounts for a high rate of relapse/recurrence, which appear to underpin the strong association between $\mathrm{BV}$ and spontaneous preterm birth.

\section{Cervicovaginal Mucosal Barrier Composition}

The mucus lining of the cervicovaginal epithelium is composed mainly of water $(\sim 95 \%)$ and mucin that accounts for its viscoelastic gel-like properties [36, 37]. Mucus also contains fatty acids, phospholipids, cholesterol, electrolytes and proteins with non-specific antimicrobial actions including immunoglobulin A, lysozyme, defensins, growth factors, lactoferrin and trefoil factors (Fig. 1) [36-38]. Cervicovaginal mucus provides a thick physical protective barrier and lubrication that prevents contact between epithelial cells and pathogens [39].

Mucins are large extracellular glycoproteins that are linked by $\alpha$-O-glycosidic linkages $[36,37]$. The terminal carbohydrate side chains protect the central protein core from degradation by proteolytic enzymes. The terminal side chains comprise mainly of sialic acid, L-fucose and sulphates, whilst the backbone contains galactose and $\mathrm{N}$-acetylglucosamine. The inner protein core consists of polypeptides with multiple tandem repeats of serine, threonine and proline residues (STP repeats) that constitute $>60 \%$ of the amino acids; and cysteine-rich regions located at the amino and carboxy terminals and occasionally intermixed between the STP repeats. The O-glycosidic linkage to the STP repeats of the protein core is through $N$-acetylgalactosamine [36-38], whilst the cysteine-rich regions contain comparatively little glycosylation [36]. More details on current understanding of the interactions between cervicovaginal microbial communities, mucus barrier and cervical mucus plug in both physiological and BV states; as well as specific mucins and their role in BV can be found in the review by Lacroix et al. [40].

\section{Degradation of Cervicovaginal Mucosal Barrier and Immunoglobulin A}

Mucins provide protection against pathogenic bacteria and fungi, and protects the upper genital tract from microbial invasion [37]. Sialic acid ( $N$-acetylneuraminic acid, Neu5Ac) in mucin inhibits bacterial adhesion to vaginal epithelial cells and biofilm formation. Hydrolysis of sialic acid terminal on the glycans of mucous membranes by sialidase facilitates adhesion of bacteria on the vaginal epithelium and formation of biofilms [37, 41, 42]. Sialidase is produced by G. vaginalis, Prevotella spp., Bacteroides fragilis, Mycoplasma hominis and Mobiluncus spp. [37, 39].

In addition to sialidase, other bacterial glucosidases degrade various carbohydrate residues of mucins [37] and expose the inner protein core to proteolysis by proteases including prolidase (proline dipeptidase) produced by $G$. vaginalis, Mobiluncus spp. and Peptostreptococcus spp. Sialidase and other glucosidases also cleave the terminal sialic acid and catalyse proteolysis of sialoglycoproteins of fibronectins and cell adhesion molecules, that also participate in mucosal barrier and immune functions [4, 43, 44]. 
Fig. 2 Plausible mechanisms of immune impairment and evasion in bacterial vaginosis teria suppress and overwhelm producing several virulent factors including mucolytic enzymes, cholesterol-dependent cytolytic toxins and short chain fatty acids that can act singly but often synergistically. AntiGvh IgA, immunoglobulin A against Gardnerella vaginalis haemolysin; $B V A B$, bacterial vaginosis-associated bacteria; Gvh, Gardnerella vaginalis haemolysin; HIV, human immunodeficiency virus; $I L$, interleukin; $\operatorname{sIg} A$, secretory immunoglobulin A; STI, sexually transmitted infection infection. BV-associated bacthe host immune response by

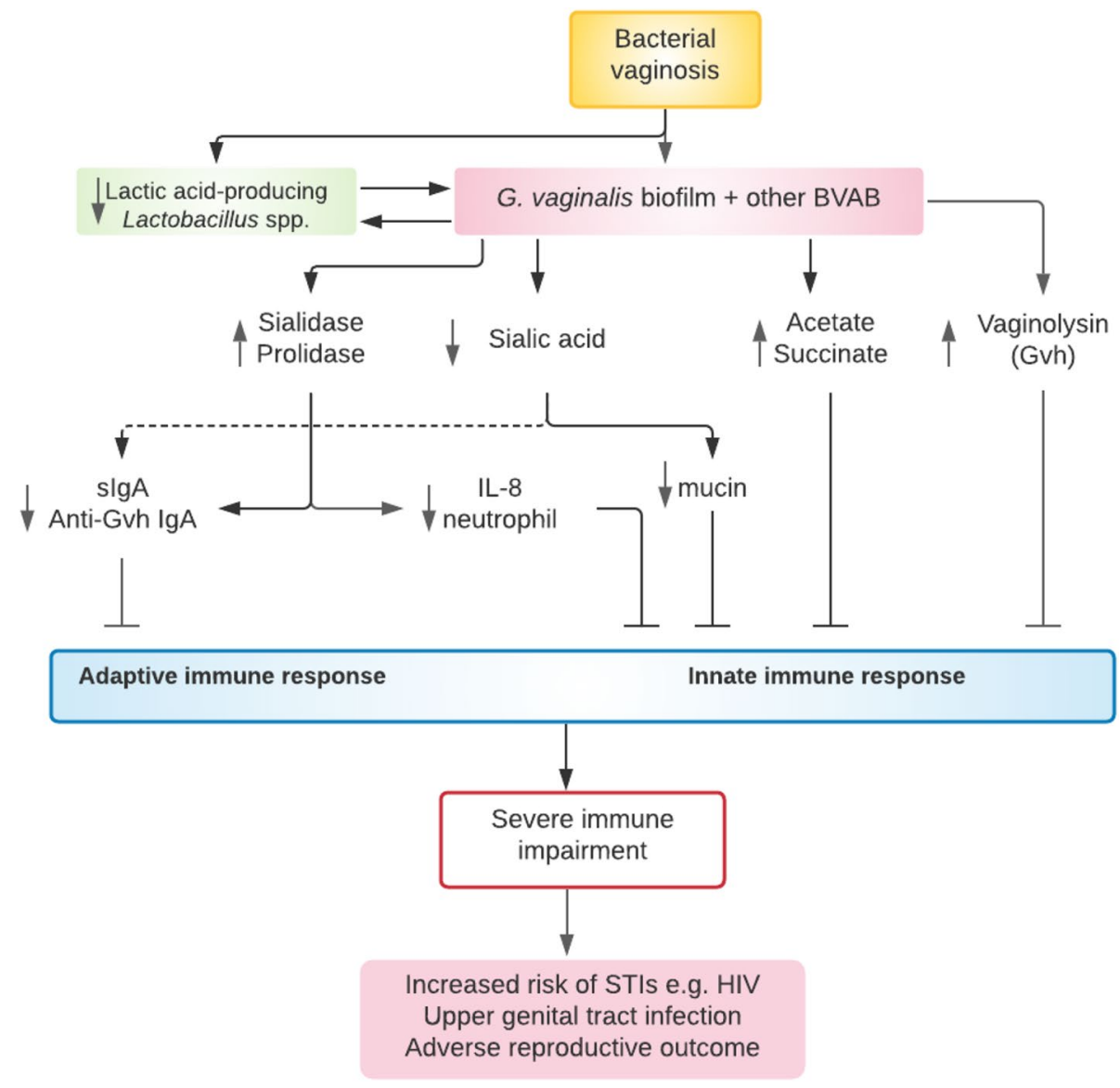

Sialidase also cleaves the terminal sialic acid from secretory $\operatorname{Ig} \mathrm{A}(\operatorname{sg} \mathrm{A})$ thereby increasing its susceptibility to proteolytic degradation $[43,45,46]$. The carbohydrate residues of $\operatorname{sg} \mathrm{A}$ are overlaid and protected by the terminal sialic acid residues. Removal of sialic acid exposes the carbohydrate residues to hydrolysis by exoglycosidases (galactosidase, glucosidase, hexosaminidase) [37,43]. Desialylation or deglycosylation of the secretory component of SIgA hampers immune response by facilitating its proteolysis [43] by proteases produced by G. vaginalis, Prevotella spp. and Ureaplasma urealyticum [37]. The non-specific antibacterial activities of sIgA are eventually compromised (Fig. 2) [46].

Furthermore, other pathways of impaired immune response and evasion adopted by $\mathrm{BV}$-associated bacteria include the enhanced activity of haemolysin (vaginolysin) secreted by $G$. vaginalis and ineffective IgA response against vaginolysin; the liberation and general depletion of mucosal sialic acid; the inhibition of cytokine-induced neutrophilic infiltration; and metabolite-induced paralysis of polymorphonuclear leukocyte chemotaxis. Other pathways also include the tolerance/resistance of BV biofilms mounted against both host defence and antimicrobial molecules (Fig. 2) and the putative action of SIGLECS are discussed in detail below.

\section{G. vaginalis Haemolysin (Vaginolysin) and Diminished IgA Response Against Vaginolysin}

G. vaginalis also obliterates the vaginal epithelial barrier function by the formation of pores on the membranes and lyses of the cells. Disrupted membrane integrity leads to cell death and loss of cellular function [47]. This virulence mechanism is perpetrated by a $G$. vaginalis haemolysin (Gvh), which is a pore-forming cholesterol-dependent cytol$y \sin (\mathrm{CDC})$ that is cytotoxic to eukaryotic cells including red blood cells $[48,49]$. In line with CDC nomenclature, the $57 \mathrm{kDa}$ toxic protein was later named vaginolysin (Fig. 2) $[47,50,51]$.

Vaginolysin is cytotoxic to vaginal epithelia cells [41], in a species-specific manner that is dependent upon the recognition of human complement regulatory glycoprotein CD59 and membrane cholesterol $[47,50]$. It is very active at high $\mathrm{pH}(5.0-7.5)$ as seen in $\mathrm{BV}$ with significantly lower levels observed when Lactobacillus crispatus dominates the microbiota [47]. Vaginolysin also induces protein kinasedependent apoptosis in human epithelial and red blood cells $[4,50]$. Through the destruction of the vaginal epithelial cells (desquamation), vaginolysin neutralises the physical 
and biochemical protection against pathogens mounted by the epithelial cells [41, 52].

In about $60 \%$ of women with BV, there is an adaptive antigen-specific immune response involving a vaginal mucosal IgA response against Gvh [23, 48, 49]. This mucosal adaptive immune response is diminished when sialidase and prolidase levels are elevated [53], with the attendant increased cleavage of vaginal fluid IgA (Fig. 2) [45]. Increased cleavage of IgA neutralises the anti-Gvh IgA protection against Gvh [53], and allows the haemolytic toxin to evade clearance and perform its cytolytic activity unabated leading to destruction and desquamation of the vaginal epithelial cells that eventually form the characteristic clue cells of BV infection [41, 49, 54]. Clue cells are formed when BV-associated bacteria such as $G$. vaginalis, Bacteroides spp. and Mobiluncus spp. are attached to exfoliated vaginal epithelial cells when lactobacilli are depleted and vaginal $\mathrm{pH}$ is greater than 4.5 [1, 55-57]. The attached bacteria release lytic enzymes such as sialidase and vaginolysin which facilitate invasion and destruction of the epithelial cells [39, 41, 58]. The host immune response is consequently suppressed [4]. High levels of anti-Gvh IgA appear protective against adverse pregnancy outcomes [23, 59]. BV-positive women with strong anti-Gvh IgA response and low sialidase and prolidase activities are not at risk of adverse pregnancy outcome such as low birth weight [60].

Taken together, the sialidase-induced deglycosylation and increased proteolysis of IgAs [43] as well as degradation of the protective mucus of vaginal epithelial barrier [4] attenuates the host IgA-mediated and innate immune responses. This possibly diminishes the ability of the female reproductive mucosa to neutralise and eliminate pathogens [43]. The integrity of the vaginal mucosal epithelium and cervical mucus is compromised, thereby facilitating ascending genital tract infection [22, 27, 39, 61].

Sialidase and $\beta$-N-acetyl-hexosaminidase are significantly increased in BV [3], and increased sialidase and/or prolidase activity is associated with preterm birth, premature rupture of membranes, low birth weight and very low birth weight [3, 4, 59-62] when combined with vaginal $\mathrm{pH}>5$ [45]. Sialidase predicted preterm delivery ( $\leq 34$ weeks) with a high specificity and positive predictive value in women with BV or intermediate microflora [45].

\section{Sialic Acid Degradation, Foraging and Depletion}

Vaginal sialidase activity is diagnostic of BV and independently correlates with risk of ascending genital tract infections and preterm birth [39]. Sialic acid-rich components of mucus such as mucin and IgA have protective and immunological functions [39]. Glycosylated mucus proteins contain about $16 \%$ sialic acid by weight [39]. To further evade host immune response, $G$. vaginalis employs sialidase to degrade and deplete vaginal mucus components containing sialic

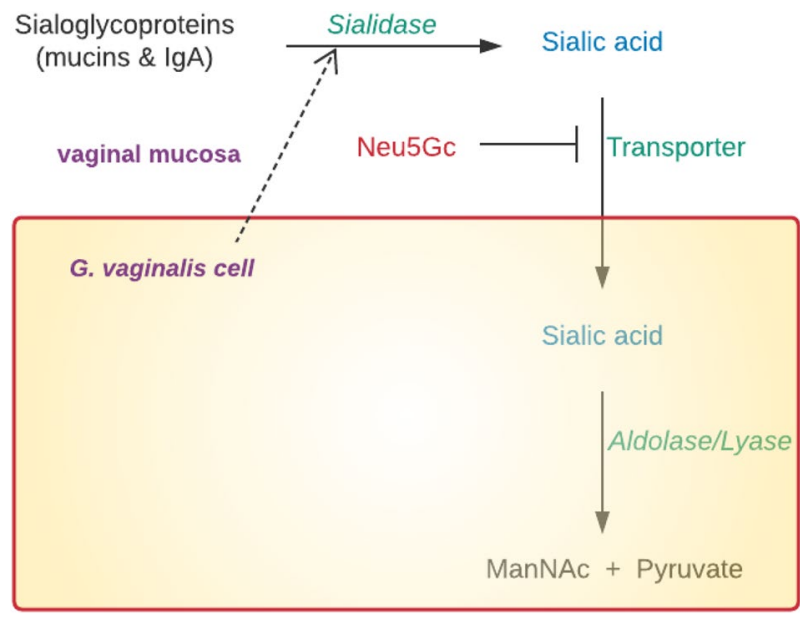

Fig. 3 Sialic acid degradation, foraging and depletion induced by G. vaginalis. Sialic acid is cleaved from mucosal sialoglycoproteins such as mucins and immunoglobulin A ( $\operatorname{Ig}$ A) that form the first line of defence against invading pathogens. The free extracellular sialic acid is then captured and ingested by $G$. vaginalis via a high-affinity transport system that can be inhibited by $N$-glycosylneuraminic acid (Neu5Gc). Intracellular sialic acid is eventually catabolised by aldolase/lyase to $\mathrm{N}$-acetylmannosamine (ManNAc) and pyruvate. This dampens the host immune response against bacterial vaginosis-associated bacteria leading to the absence of an obvious inflammation and increased risk of adverse reproductive outcomes

acid. Extracellular sialidase hydrolyses mucosal sialoglycoproteins and the cleaved sialic acid is transported by a highaffinity transport system into the bacterium. This is an active process enhanced by glucose but inhibited by excess $\mathrm{N}$-glycosylneuraminic acid (Neu5Gc). The ingested sialic acid is then catabolised by intracellular aldolase/lyase reaction to $\mathrm{N}$-acetylmannosamine (ManNAc) and pyruvate (foraging) (Fig. 3) [39]. Sialic acid catabolism provides nutrients that support bacterial growth [63]. This sialidase activity, which is not observed in healthy controls, i.e. women with healthy lactobacilliary vaginal microbiota [39, 43], leads to significantly reduced vaginal sialic acid in women with BV [39].

Depletion of the components of the protective mucosal barrier facilitates microbial adhesion and invasion of the upper genital tract $[1,39]$. Though the vaginal sialidase activity and sialic acid foraging have been characterised in G. vaginalis extensively, other BV-associated bacteria such as Bacteroides spp. and Prevotella spp. can also produce the same pathogenic effect [39]. Future studies can determine the association of vaginal bacterial composition, level of sialidase activity and degree of sialic acid depletion in pregnant and non-pregnant women. The possibility of these variables singly or in combination to distinguish women at risk of preterm birth or other adverse reproductive outcomes can also be investigated. 


\section{Inhibition of IL-8-Induced Neutrophilic Infiltration}

The production of sialidase and prolidase by $\mathrm{BV}$-associated bacteria is associated with increased cervicovaginal IL- $1 \beta$. In spite of the increase in IL- $1 \beta$ levels, there is no concomitant increase in the neutrophil chemoattractant and activator, IL-8 [23, 64]. Though IL- $1 \beta$ promotes IL-8 secretion, IL-8 concentration and vaginal neutrophils are low in women with BV $[64,65]$. The bacterial hydrolytic enzymes downregulates the IL- $8 / \mathrm{IL}-1 \beta$ ratio thereby blunting the IL- $1 \beta$ induced proinflammatory cascade leading to reduction in neutrophil infiltration typical of BV [64].

IL-8 levels were inversely correlated with elevated sialidase and prolidase, and positively associated with anti-Gvh IgA response and neutrophils in women with BV [23, 53, $64]$, i.e. both innate and adaptive immune responses are impaired in BV [44]. In vivo experiments have shown that $\mathrm{BV}$-associated bacteria can circumvent immune response by inhibiting IL-8 production and stability or secrete factors that facilitate IL-8 degradation [2]. The obvious decrease in vaginal neutrophils is the hallmark of $\mathrm{BV}$, which indicates suppressed innate mucosal immunity (Fig. 2) [65].

Sialidase and prolidase can also degrade cationic antimicrobial polypeptides (cAMPs) such as human $\beta$ defensins 1 and 2, secretory leukocyte protease inhibitor (SLPI) [64]; lactoferrin, some cytokines and cellular receptors such as Toll-like receptors (TLR) 2 and 4 [23, 66], which are the main effector molecules of vaginal innate and adaptive mucosal immunity against pathogens $[1,65]$. The cAMPs are also engage in protection against the proteolytic enzymes released by pathogenic bacteria, fungi and some viruses as well as direct microbial killing [52]. Therefore, degradation of these protective molecules impairs the immune response and may increase the risk of poor reproductive outcomes in pregnant women with BV [64].

\section{Incapacitation of Neutrophil and Monocyte Chemotaxis}

Neutrophil and monocyte chemotaxis is vital to a potent innate immune response to pathogenic stimuli. BV-associated bacteria metabolise carbon sources in the vaginal environment to short chain volatile fatty acids (SCFAs) including acetate, butyrate, isobutyrate, propionate and the non-volatile fatty acid-succinate. These metabolites increase vaginal $\mathrm{pH}$ that permits the colonisation of the cervicovaginal space by $\mathrm{BV}$-associated bacteria and predispose the host to infection [1]. In addition to this already deleterious phonotype, the metabolites especially acetate and succinate are capable of inhibiting chemotaxis of immunocompetent cells including neutrophils and monocytes $[67,68]$, thereby incapacitating the host response against invading pathogens, and promoting infection and adverse reproductive outcomes such as preterm birth [25] (Fig. 2). For instance, succinate inhibits phagocytosis of $E$. coli as well as neutrophil chemotaxis. This anti-chemotactic action helps the infectious agents to dodge phagocytosis and the resultant neutrophil activity, and could account for the absence of pus cells (polymorphonuclear leukocytes) in vaginal secretions of women with BV [67]. The evasion of phagocytosis is usually the first action by pathogens in the induction of any infection including purulent (pyogenic) infections. The next action is prolong inhibition of neutrophil and monocyte chemotaxis which permits growth, proliferation and colonisation, and infection is established [67].

Succinate appears to be more potent than acetate in chemotaxis inhibition. However, both metabolites may act synergistically to amplify the anti-chemotaxis observed in $\mathrm{BV}$. Lactic acid that is predominant in the cervicovaginal fluid of healthy women does not exhibit this inhibitory effect. In vitro experiments have shown that supernatants of Prevotella spp. and Mobiluncus spp. produced significantly higher succinate and acetate, thus, more anti-chemotatic effect compared to Gardnerella spp. Succinate and acetate are believed to disrupt the binding of chemotactic factor to the surface of polymorphonuclear leukocytes. Some Prevotella spp. have also been reported to produce succinate and acetate that inhibit chemotaxis of leukocytes irreversibly [67].

Because $\mathrm{BV}$ is a polymicrobial dysbiotic condition, the mixed microbiota could act in synergy, i.e. each organism amplifying or potentiating the virulence mechanism of the other, thereby contributing to the overall evasion of host immune response [67]. For instance, Prevotella spp. and Mobiluncus spp. could produce more succinate and acetate to incapacitate neutrophils [67] allowing G. vaginalis ample time to proliferate and form biofilms that are resistant to the antimicrobial and anti-inflammatory effects of lactic acid-producing lactobacilli, mucosal defences and antibiotics. The G. vaginalis biofilm in turn provide a scaffold for the build-up of other BV-associated bacteria [41, 69]. This produces a more formidable resistance in addition to evading the host immune response resulting in the persistence and recurrence of BV. This synergistic biofilm formation effect between $\mathrm{BV}$-associated bacteria can also be achieved via sialidase and vaginolysin-mediated mucosal/innate defence impairment [41]. The polymicrobial synergistic interaction linked to the pathomechanism of $\mathrm{BV}$ is established as $G$. vaginalis is present in nearly $90 \%$ of women without BV and as such may not be the sole causative organism in all instances [70].

\section{Polymicrobial Biofilm Formation}

A biofilm is a community of microorganisms encapsulated in a polymeric matrix of polysaccharides, proteins and nucleic 
acids, and attached to a surface [71]. The stages of biofilm formation include the following: (i) adhesion, (ii) microcolony formation and coaggregation, (iii) maturation and (iv) dispersion (erosion and sloughing induced by hydrolytic enzymes or increased $\mathrm{pH}$ in the vagina) [7]. Bacteria within biofilms are usually shielded from the host immune response and antibiotic therapy resulting in the persistence of such infections. Standard antibiotics such as metronidazole are unable to completely clear BV vaginal biofilms-associated bacteria [72]. For example, oral metronidazole therapy on adherent $G$. vaginalis biofilms only temporarily suppressed the biofilms, which immediately resumed pathogenic activity after treatment cessation [73]. BV biofilm-forming bacteria also exhibited resistance to metronidazole, tinidazole and clindamycin in an in vitro study [74]. In this study [74], G. vaginalis had the greatest virulence capacity indicated by higher initial adhesion and cytotoxicity of epithelial cells, as well as greater tendency to form a biofilm compared to other biofilm-forming BV-associated bacterial species including Streptococcus agalactiae, Gemella haemolysans, Enterococcus faecalis, Propionibacterium acnes, Mycoplasma hominis and Escherichia coli. [74]. Therefore, biofilm formation also accounts for the high rate of relapse and recurrence seen in BV cases [72-74]. More than 50\% of women treated for BV will have recurrent episode(s) within 6-12 months [75]. That is, in addition to the overgrowth of anaerobes, BV is associated with the presence of a dense, structured and adherent polymicrobial biofilm assembled by $G$. vaginalis on the vaginal mucosa [76].

It is believed that the initiation and progression of $\mathrm{BV}$ is dependent/induced by the formation of $G$. vaginalis biofilm $[72,76]$, which can re-form following oral metronidazole treatment in some instances [73], as it serves as reservoir for regrowth of pathogens [77]. G. vaginalis also produces extracellular DNA (eDNA) that stimulates the formation of extracellular polymeric substance matrix implicated in biofilm maturation and persistence [7, 78]. This initial biofilm serves as the scaffold for the coaggregation of other BVassociated anaerobes such as Atopobium vaginae, Prevotella bivia, Mobiluncus mulieris, Fusobacterium nucleatum and Peptoniphilus spp. [7, 30, 41, 73, 76, 79-85], thereby forming an intractable virulent polymicrobial alliance attached tightly to the surface of the vaginal epithelium like a "brickwork"[76], and persistently expressing destructive immune suppressing molecules such as hydrolytic enzymes, vaginolysin [41, 72, 73] and SCFAs [1, 25]. Other BV-related species including Bacteroides spp., Streptococcus spp., Veillonella spp., [76], E. coli, E. faecalis and Actinomyces neuii $[7,41,84,86]$ have also been found on biofilms augmenting the growth of $G$. vaginalis. G. vaginalis serves an early coloniser in the development of multispecies biofilm, whilst the other BV-associated species serve as second/third colonisers [7]; although the process may be initiated by Peptoniphilus spp. [87] instead of G. vaginalis. The extracellular polymeric matrix produced by the adherent bacterial species encapsulates and shields the biofilm from host immune system and antibiotics [72].

By releasing sialic acid from mucosal sialoglycans, sialidase unmasks the cryptic host ligands required for bacterial adherence. This enhances biofilm formation, bacterial colonisation [37, 41, 42, 63], loss of membrane integrity and damage to epithelial cells $[47,88]$. The cytotoxic action of vaginolysin also contributes to vaginal epithelial cell desquamation that manifest as the characteristic clue cells of BV [39, 41, 55, 57, 58]. A. neuii or E. faecalis when colonising biofilms formed by $G$. vaginalis can stimulate overexpression of vaginolysin and sialidase genes by $G$. vaginalis cells to facilitate formation of clue cells [41]. By contrast, healthpromoting $L$. crispatus represses vaginolysin expression by G. vaginalis and reduces vaginal epithelial cell cytotoxity $[47,89]$. However, G. vaginalis still adheres to vaginal epithelial cells in the presence of $L$. crispatus with the assistance of Lactobacillus iners [82, 90] and Peptoniphilus spp. $[79,82,87]$. Biofilms play vital roles in the pathogenesis of $\mathrm{BV}$ mounting tolerance or resistance to the host-microbial defence mechanisms of normal vaginal microbiome including lactic acid, $\mathrm{H}_{2} \mathrm{O}_{2}$, mucosal immune defences, antibodies, as well as demonstrating enhanced antibiotic tolerance $[4$, $41,72,73,91]$.

$\mathrm{BV}$-associated pathogens also produce other substances that target B-cells and immunoglobulins such as haemolysin III, superantigen (L-protein) and peptostreptococcal albumin-binding proteins [4], and suppress the host immunity. When these BV-associated organisms form a biofilm with each demonstrating its virulence as well as immune suppressing or subversion properties, there is rarely a noticeable inflammatory response. Hence, established but uncomplicated BV is typically characterised by lack of leukocytes on microscopy, the vagina is not inflamed or reddish, and no significant burning sensation, pain or dyspareunia is observed [34].

\section{SIGLEC-Induced Negative Regulation of Immune Response}

The lipopolysaccharide (LPS) receptor complex comprise of pattern recognition receptors (PRRs) that are sialylated glycoconjugates, i.e. TLR4, myeloid differentiation protein 2 (MD-2) and CD14. CD14 is a glycosylphosphatidylinositolanchored glycoprotein expressed on leukocytes, which facilitates binding of LPS to TLR4. LPS/TLR4 binding initiates $\mathrm{NF}-\mathrm{kB}-$ mediated proinflammatory cytokine production [92]. Elevated sialidase activity as seen during immune cell differentiation/activation [93] and BV [3], cleaves sialic acid from PRRs on the surface of immune cells [23, 66, 93]. 
Table 2 Immune classification of women with bacterial vaginosis

\begin{tabular}{lll}
\hline $\begin{array}{l}\text { Low risk } \\
\text { Maintained local immune response }\end{array}$ & $\begin{array}{l}\text { High risk } \\
\text { Impaired immune response }\end{array}$ & References \\
\hline Low cleavage of IgA & Increased cleavage of IgA & {$[23,46,54]$} \\
Low sialidase and prolidase activity & High sialidase and prolidase activity & {$[23,46,54]$} \\
Positive anti-Gvh IgA response & No anti-Gvh IgA response & {$[23,46,54]$} \\
Increased IL-8 and neutrophil chemotaxis & Reduced IL-8 and neutrophil chemotaxis & {$[23,46,54]$} \\
\hline
\end{tabular}

Anti-Gvh IgA, immunoglobulin A against Gardnerella vaginalis haemolysin; Gvh, Gardnerella vaginalis haemolysin
Sialic acid removal facilitates response of the PRRs to LPS and production of proinflammatory cytokines [93].

However, inhibitory receptors such as sialic acid-binding immunoglobulin-like lectins (SIGLECS) expressed by immune cells attenuate the expected immune reaction by binding to the sialylated glycoconjugates (PRRs) [94]. For instance, CD33 (Siglec-3), the smallest member of the SIGLEC family, down-regulates TLR4 mediated signalling by binding to its cis ligand CD14. The CD14/CD33 interaction regulates the presentation of LPS from CD14 to TLR4, thereby down-regulating the LPS-NF- $\mathrm{KB}$ proinflammatory pathway [92]. It is plausible that this SIGLEC-mediated negative regulation of TLR4 signalling may contribute to the attenuation of pro-inflammatory response observed in $\mathrm{BV}$. This warrants further investigation.

\section{Future Perspectives}

The lack of an appropriate inflammatory response in BV despite obvious colonisation of the lower genital tract by pathogenic bacteria resulting from disruption of vaginal homeostasis has been a great concern to clinicians and researchers. This has greatly impacted early diagnosis and prompt treatment of women with the condition. Hence, recurrent $\mathrm{BV}$ remains a major public health burden especially in the pregnant population.

The molecular mechanisms of immune evasion in BV have been studied in isolation leading to absence of a comprehensive understanding and report of the factors that distinguish BV from vaginitis and other female genital tract infections. This is a critical review that attempts to discuss and link the probable mechanistic characteristics underpinning the enigmatic and precarious suppression and subversion of host immune system/response by BV-associated bacteria.

We observe that BV incorporates a spectrum of alterations including increased species biodiversity, production of immunomodulatory enzymes and metabolites, and suboptimal host biochemical and immunological responses [23, 45]. That is, it is not only a decrease in lactic acid-producing Lactobacillus spp. and overgrowth of mixed anaerobes, but also increase in mucin-degrading enzymes (e.g. sialidase and prolidase), acetate and succinate, and cytotoxins such as vaginolysin that characterise BV. This deleterious phenotype is aggravated when the organisms form biofilms that confer additional resistance to host defence and antibiotic clearance. The host response is characterised by reduced IL-8 levels and neutrophil infiltration. Therefore, the host-microbial interactions at the vaginal mucosa is pivotal to the sequelae of $\mathrm{BV}$ infection.

Though the underlying mechanistic factors can act synergistically, the impact of each of them differ amongst patients, and this is crucial for accurate diagnosis and tailored therapeutic interventions to preclude relapse and recurrence. Of these factors, sialidase appears to be central to the mechanistic pathways and has attracted more research attention, being employed as a diagnostic test for BV (BVBlue test) [95, 96]. Sialidase degrades sialic acid and by extension disrupts mucosal epithelial membranes and facilitates bacterial attachment, biofilm formation and IgA degradation. This sets the stage for the continuous production and activities of other virulence factors including vaginolysin and SCFAs. Elevated levels of sialidase are associated with preterm birth and low birth weight, with modest predictive capacity for preterm birth [3, 4, 59-62]. Elevated acetate levels have also predicted preterm birth especially in women presenting with symptoms of preterm labour [97-99]. Whether sialidase alone or in combination with other microbial virulence and/or host immune factors is clinically useful for the diagnosis of preterm birth and related poor pregnancy outcomes remains to be established and therefore necessitates further investigation.

Because of the immunosuppression and subversion of the immune response, BV women show highly variable clinical prognosis. Some BV infected women recover without treatment, some harbour the infection without symptoms for years especially when poorly treated, others recover after a single treatment, whilst others require multiple or continuous treatments. Consequently, women with BV can be subclassified into those that maintain local immune response and those with impaired immune response (Table 2) [46, 54]. BV-positive women that maintain local immune response show less aggressive disease and better prognosis. In contrast, those with compromised immune response induced mostly by high sialidase exhibit a more aggressive disease 
and are at greater risk of relapse and recurrence even though they are often asymptomatic. This sub-classification should improve treatment of BV-positive women [23].

Furthermore, the possibility of targeting (inhibiting) sialidase activity for therapeutic purposes can rehabilitate the dysbiotic vaginal microbiota to a lactobacilliary (eubiotic) status by suppressing sialidase-positive BV bacteria. As demonstrated in mice models, this would reduce the level of free sialic acid and starve BV pathogens such as group B Streptococcus of key nutrients, and prevent ascending genital tract infection and chorioamnionitis [88]. The anti-influenza drug Zanamivir has shown ability to inhibit sialidase activity and invasion of human cells by $G$. vaginalis. This can reduce bacterial adhesion and inflammation by maintaining functional IgA [43]. Some of our colleagues (Galleh et al.) [100] have also explored the effectiveness of plant-derived (e.g. Epicatechin gallate and Berberine chloride) and synthetic (2,3-Difluorosialic acid analogues) sialidase inhibitors compared to Zanamivir in the oral cavity. Further testing of the anti-sialidase activity of Zanamavir and the oral sialidase inhibitors in a polymicrobial vaginal microbiota model is imperative.

\section{Conclusion}

In conclusion, sialidase is central to the immunosuppression observed in BV that accounts for the absence of an overt inflammatory response, many asymptomatic cases and high rate of relapse and recurrence. Its pathogenic effects are supported by other hydrolytic enzymes, haemolytic toxins and immunomodulatory cytokines and metabolites. All these factors are produced or induced by several BV-associated bacteria with $G$. vaginalis as the prime culprit in most cases. Although sialidase and acetate have shown capacity to identify women at risk of preterm birth, further studies are required to validate their clinical utility independently or combined with the other virulence factors. Such studies can be conducted in women of different ethno-racial groups and/or living in different geographic locations. Finally, if the natural and/or synthetic sialidase inhibitors are effective as therapeutic (eubiotic) agents in the vaginal milieu, they could potentially reduce the incidence of recurrent $\mathrm{BV}$ in the general and pregnant populations as well as the excessive use of antibiotics and antibiotic resistance.

Acknowledgements NIHR Global Health Research Group on PReterm bIrth prevention and manageMEnt (PRIME) at The University of Sheffield, UK; University of Cape Town, South Africa; University of Pretoria, South Africa; and The International Centre for Diarrhoeal Disease Research (icddr,b), Bangladesh. https://www.primeglobalhealth.co.uk

Author Contribution Conceptualization and literature search-EA; original draft preparation, review and editing-EA and DA. Both authors approved the final draft of the manuscript for submission.
Funding EA and DA are funded by National Institute for Health Research (NIHR, 17/63/26).

Availability of Data and Material Not applicable.

Code Availability Not applicable.

\section{Declarations}

Conflict of interest The authors have no relevant financial or non-financial interests to disclose.

Ethical Approval This review article is part of larger project approved by the HRA and Health and Care Research Wales (HCRW, REC reference: $18 / \mathrm{LO} / 2044)$.

Consent to Participate Not applicable.

Consent for Publication Not applicable.

Open Access This article is licensed under a Creative Commons Attribution 4.0 International License, which permits use, sharing, adaptation, distribution and reproduction in any medium or format, as long as you give appropriate credit to the original author(s) and the source, provide a link to the Creative Commons licence, and indicate if changes were made. The images or other third party material in this article are included in the article's Creative Commons licence, unless indicated otherwise in a credit line to the material. If material is not included in the article's Creative Commons licence and your intended use is not permitted by statutory regulation or exceeds the permitted use, you will need to obtain permission directly from the copyright holder. To view a copy of this licence, visit http://creativecommons.org/licenses/by/4.0/.

\section{References}

1. Amabebe E, Anumba DOC (2018) The vaginal microenvironment: the physiologic role of Lactobacilli. Front Med 5:181. https://doi.org/10.3389/fmed.2018.00181

2. Santos CMA, Pires MCV, Leão TL, Silva AKS, Miranda LS, Martins FS et al (2018) Anti-inflammatory effect of two Lactobacillus strains during infection with Gardnerella vaginalis and Candida albicans in a HeLa cell culture model. Microbiology (Reading) 164(3):349-358. https://doi.org/10.1099/mic.0.000608

3. Jefferson KK (2012) The bacterial etiology of preterm birth. Adv Appl Microbiol 80:1-22. https://doi.org/10.1016/b978-012-394381-1.00001-5

4. Basavaprabhu HN, Sonu KS, Prabha R (2020) Mechanistic insights into the action of probiotics against bacterial vaginosis and its mediated preterm birth: an overview. Microb Pathog 141:104029. https://doi.org/10.1016/j.micpath.2020.104029

5. Ramos Bde A, Kanninen TT, Sisti G, Witkin SS (2015) Microorganisms in the female genital tract during pregnancy: tolerance versus pathogenesis. Am J Reprod Immunol 73(5):383-389. https://doi.org/10.1111/aji.12326

6. Morrill S, Gilbert NM, Lewis AL (2020) Gardnerella vaginalis as a cause of bacterial vaginosis: appraisal of the evidence from in vivo models. Front Cell Infect Microbiol 10:168. https://doi. org/10.3389/fcimb.2020.00168

7. Jung HS, Ehlers MM, Lombaard H, Redelinghuys MJ, Kock MM (2017) Etiology of bacterial vaginosis and polymicrobial biofilm formation. Crit Rev Microbiol 43(6):651-667. https://doi.org/10. 1080/1040841x.2017.1291579 
8. Srinivasan S, Morgan MT, Fiedler TL, Djukovic D, Hoffman NG, Raftery D et al (2015) Metabolic signatures of bacterial vaginosis. mBio. 6(2):e00204-e00215. https://doi.org/10.1128/ mBio.00204-15

9. Mohamed I, Zakeer S, Azab M, Hanora A (2020) Changes in vaginal microbiome in pregnant and nonpregnant women with bacterial vaginosis: toward microbiome diagnostics? OMICS J Integr Biol 24(10):602-14. https://doi.org/10.1089/omi.2020. 0096

10. Schwebke JR, Muzny CA, Josey WE (2014) Role of Gardnerella vaginalis in the pathogenesis of bacterial vaginosis: a conceptual model. J Infect Dis 210(3):338-343. https://doi.org/10.1093/ infdis/jiu089

11. Schwebke JR, Muzny CA, Josey WE (2014) Reply to Hickey and Forney. J Infect Dis 210(10):1683-1684. https://doi.org/10.1093/ infdis/jiu304

12. Hickey RJ, Forney LJ (2014) Gardnerella vaginalis does not always cause bacterial vaginosis. J Infect Dis 210(10):16821683. https://doi.org/10.1093/infdis/jiu303

13. Muzny CA, Taylor CM, Swords WE, Tamhane A, Chattopadhyay D, Cerca N et al (2019) An updated conceptual model on the pathogenesis of bacterial vaginosis. J Infect Dis 220(9):1399_ 1405. https://doi.org/10.1093/infdis/jiz342

14. Balashov SV, Mordechai E, Adelson ME, Gygax SE (2014) Identification, quantification and subtyping of Gardnerella vaginalis in noncultured clinical vaginal samples by quantitative PCR. J Med Microbiol 63(2):162-175. https://doi.org/10.1099/jmm.0. 066407-0

15. Cox C, McKenna JP, Watt AP, Coyle PV (2015) New assay for Gardnerella vaginalis loads correlates with Nugent scores and has potential in the diagnosis of bacterial vaginosis. J Med Microbiol 64(9):978-984. https://doi.org/10.1099/jmm.0.000118

16. Ceccarani C, Foschi C, Parolin C, D'Antuono A, Gaspari V, Consolandi $\mathrm{C}$ et al (2019) Diversity of vaginal microbiome and metabolome during genital infections. Sci Rep 9(1):14095. https://doi.org/10.1038/s41598-019-50410-x

17. Amabebe E (2016) Analysis of cervicovaginal fluid metabolome microbiome in relation to preterm birth. $\mathrm{PhD}$ thesis, University of Sheffield White Rose eTheses Online, WREO. 2016;13862. http://etheses.whiterose.ac.uk/13862/.

18. Amabebe E, Anumba DOC (2018) Psychosocial stress, cortisol levels, and maintenance of vaginal health. Front Endocrinol 9:568. https://doi.org/10.3389/fendo.2018.00568

19. Peebles K, Velloza J, Balkus JE, McClelland RS, Barnabas RV (2019) High global burden and costs of bacterial vaginosis: a systematic review and meta-analysis. Sex Transm Dis 46(5):304311. https://doi.org/10.1097/olq.0000000000000972

20. Redelinghuys MJ, Geldenhuys J, Jung H, Kock MM (2020) Bacterial vaginosis: current diagnostic avenues and future opportunities. Front Cell Infect Microbiol 10:354. https://doi.org/10.3389/ fcimb. 2020.00354

21. Deb K, Chaturvedi MM, Jaiswal YK (2004) Comprehending the role of LPS in Gram-negative bacterial vaginosis: ogling into the causes of unfulfilled child-wish. Arch Gynecol Obstet 270(3):133-146. https://doi.org/10.1007/s00404-004-0623-0

22. Ravel J, Moreno I, Simón C (2021) Bacterial vaginosis and its association with infertility, endometritis, and pelvic inflammatory disease. Am J Obstet Gynecol 224(3):251-257. https://doi. org/10.1016/j.ajog.2020.10.019

23. Cauci S (2004) Vaginal immunity in bacterial vaginosis. Curr Infect Dis Rep 6(6):450-456. https://doi.org/10.1007/ s11908-004-0064-8

24. Ranjit E, Raghubanshi BR, Maskey S, Parajuli P (2018) Prevalence of bacterial vaginosis and its association with risk factors among nonpregnant women: a hospital based study. Int J Microbiol 2018:8349601. https://doi.org/10.1155/2018/8349601
25. Amabebe E, Anumba DOC (2020) Female gut and genital tract microbiota-induced crosstalk and differential effects of shortchain fatty acids on immune sequelae. Front Immunol 11:2184. https://doi.org/10.3389/fimmu.2020.02184

26. Srinivasan S, Hoffman NG, Morgan MT, Matsen FA, Fiedler TL, Hall RW et al (2012) Bacterial communities in women with bacterial vaginosis: high resolution phylogenetic analyses reveal relationships of microbiota to clinical criteria. PLoS ONE 7(6):e37818. https://doi.org/10.1371/journal.pone.00378 18

27. Amabebe E (2016) Analysis of cervicovaginal fluid metabolome microbiome in relation to preterm birth. $\mathrm{PhD}$ thesis, University of Sheffield White Rose eTheses Online, WREO. 2016;13862, http://etheses.whiterose.ac.uk/13862/

28. Danielsson D, Teigen PK, Moi H (2011) The genital econiche: focus on microbiota and bacterial vaginosis. Ann N Y Acad Sci 1230:48-58. https://doi.org/10.1111/j.1749-6632.2011.06041.x

29. Verstraelen H, Swidsinski A (2019) The biofilm in bacterial vaginosis: implications for epidemiology, diagnosis and treatment: 2018 update. Curr Opin Infect Dis 32(1):38-42. https://doi.org/ 10.1097/qco.0000000000000516

30. Swidsinski A, Loening-Baucke V, Mendling W, Dörffel Y, Schilling J, Halwani $Z$ et al (2014) Infection through structured polymicrobial Gardnerella biofilms (StPM-GB). Histol Histopathol 29(5):567-587. https://doi.org/10.14670/hh-29.10.567

31. Barraud N, Kjelleberg S, Rice SA (2015) Dispersal from microbial biofilms. Microbiol Spectr. https://doi.org/10.1128/micro biolspec.MB-0015-2014

32. Chua SL, Liu Y, Yam JKH, Chen Y, Vejborg RM, Tan BGC et al (2014) Dispersed cells represent a distinct stage in the transition from bacterial biofilm to planktonic lifestyles. Nat Commun 5(1):4462. https://doi.org/10.1038/ncomms5462

33. Kragh KN, Hutchison JB, Melaugh G, Rodesney C, Roberts AE, Irie $\mathrm{Y}$ et al (2016) Role of multicellular aggregates in biofilm formation. mBio 7(2):e00237. https://doi.org/10.1128/mBio. 00237-16

34. Donders GG, Vereecken A, Bosmans E, Dekeersmaecker A, Salembier G, Spitz B (2002) Definition of a type of abnormal vaginal flora that is distinct from bacterial vaginosis: aerobic vaginitis. BJOG 109(1):34-43. https://doi.org/10.1111/j.14710528.2002.00432.x

35. Beamer MA, Austin MN, Avolia HA, Meyn LA, Bunge KE, Hillier SL (2017) Bacterial species colonizing the vagina of healthy women are not associated with race. Anaerobe 45:40-43. https:// doi.org/10.1016/j.anaerobe.2017.02.020

36. Bansil R, Turner BS (2006) Mucin structure, aggregation, physiological functions and biomedical applications. Curr Opin Colloid Interface Sci 11(2):164-170. https://doi.org/10.1016/j.cocis. 2005.11.001

37. Wiggins R, Hicks SJ, Soothill PW, Millar MR, Corfield AP (2001) Mucinases and sialidases: their role in the pathogenesis of sexually transmitted infections in the female genital tract. Sex Transm Infect 77(6):402-408. https://doi.org/10.1136/sti.77.6. 402

38. Pinzón Martín S, Seeberger PH, Varón Silva D (2019) Mucins and pathogenic mucin-like molecules are immunomodulators during infection and targets for diagnostics and vaccines. Front Chem 7:710. https://doi.org/10.3389/fchem.2019.00710

39. Lewis WG, Robinson LS, Gilbert NM, Perry JC, Lewis AL (2013) Degradation, foraging, and depletion of mucus sialoglycans by the vagina-adapted Actinobacterium Gardnerella vaginalis. J Biol Chem 288(17):12067-12079. https://doi.org/ 10.1074/jbc.M113.453654

40. Lacroix G, Gouyer V, Gottrand F, Desseyn J-L (2020) The Cervicovaginal Mucus Barrier. Int J Mol Sci 21(21):8266. https:// doi.org/10.3390/ijms21218266 
41. Castro J, Machado D, Cerca N (2019) Unveiling the role of Gardnerella vaginalis in polymicrobial Bacterial Vaginosis biofilms: the impact of other vaginal pathogens living as neighbors. ISME J 13(5):1306-1317. https://doi.org/10.1038/s41396-018-0337-0

42. Hardy L, Jespers V, Van den Bulck M, Buyze J, Mwambarangwe $\mathrm{L}$, Musengamana $\mathrm{V}$ et al (2017) The presence of the putative Gardnerella vaginalis sialidase A gene in vaginal specimens is associated with bacterial vaginosis biofilm. PLoS ONE 12(2):e0172522. https://doi.org/10.1371/journal.pone. 0172522

43. Lewis WG, Robinson LS, Perry J, Bick JL, Peipert JF, Allsworth JE et al (2012) Hydrolysis of secreted sialoglycoprotein immunoglobulin A ( $\operatorname{Ig} \mathrm{A})$ in ex vivo and biochemical models of bacterial vaginosis. J Biol Chem 287(3):2079-2089. https://doi. org/10.1074/jbc.M111.278135

44. Cauci S, Driussi S, Guaschino S, Isola M, Quadrifoglio F (2002) Correlation of local interleukin-1beta levels with specific IgA response against Gardnerella vaginalis cytolysin in women with bacterial vaginosis. Am J Reprod Immunol 47(5):257-264. https://doi.org/10.1034/j.1600-0897.2002.01096.x

45. Cauci S, McGregor J, Thorsen P, Grove J, Guaschino S (2005) Combination of vaginal $\mathrm{pH}$ with vaginal sialidase and prolidase activities for prediction of low birth weight and preterm birth. Am J Obstet Gynecol 192(2):489-496. https://doi.org/10.1016/j. ajog.2004.07.023

46. Cauci S, Driussi S, Monte R, Lanzafame P, Pitzus E, Quadrifoglio F (1998) Immunoglobulin A response against Gardnerella vaginalis hemolysin and sialidase activity in bacterial vaginosis. Am J Obstet Gynecol 178(3):511-515. https://doi.org/10.1016/ s0002-9378(98)70430-2

47. Pleckaityte M (2019) Cholesterol-dependent cytolysins produced by vaginal bacteria: certainties and controversies. Front Cell Infect Microbiol 9:452. https://doi.org/10.3389/fcimb.2019. 00452

48. Cauci S, Monte R, Ropele M, Missero C, Not T, Quadrifoglio $F$ et al (1993) Pore-forming and haemolytic properties of the Gardnerella vaginalis cytolysin. Mol Microbiol 9(6):1143-1155. https://doi.org/10.1111/j.1365-2958.1993.tb01244.x

49. Cauci S, Scrimin F, Driussi S, Ceccone S, Monte R, Fant L et al (1996) Specific immune response against Gardnerella vaginalis hemolysin in patients with bacterial vaginosis. Am J Obstet Gynecol 175(6):1601-1605. https://doi.org/10.1016/s00029378(96)70112-6

50. Gelber SE, Aguilar JL, Lewis KLT, Ratner AJ (2008) Functional and phylogenetic characterization of Vaginolysin, the humanspecific cytolysin from Gardnerella vaginalis. J Bacteriol 190(11):3896-3903. https://doi.org/10.1128/JB.01965-07

51. Randis TM, Kulkarni R, Aguilar JL, Ratner AJ (2009) Antibodybased detection and inhibition of Vaginolysin, the Gardnerella vaginalis cytolysin. PLoS ONE 4(4):e5207. https://doi.org/10. 1371/journal.pone.0005207

52. Al-Nasiry S, Ambrosino E, Schlaepfer M, Morré SA, Wieten L, Voncken JW et al (2020) The interplay between reproductive tract microbiota and immunological system in human reproduction. Front Immunol 11:378. https://doi.org/10.3389/fimmu. 2020.00378

53. Cauci S, Guaschino S, Driussi S, De Santo D, Lanzafame P, Quadrifoglio F (2002) Correlation of local interleukin-8 with immunoglobulin A against Gardnerella vaginalis hemolysin and with prolidase and sialidase levels in women with bacterial vaginosis. J Infect Dis 185(11):1614-1620. https://doi.org/10.1086/ 340417

54. Cauci S, Monte R, Driussi S, Lanzafame P, Quadrifoglio F (1998) Impairment of the mucosal immune system: IgA and IgM cleavage detected in vaginal washings of a subgroup of patients with bacterial vaginosis. J Infect Dis 178(6):1698-1706. https:// doi.org/10.1086/314505

55. Cook RL, Reid G, Pond DG, Schmitt CA, Sobel JD (1989) Clue cells in bacterial vaginosis: immunofluorescent identification of the adherent gram-negative bacteria as Gardnerella vaginalis. J Infect Dis 160(3):490-496. https://doi.org/10.1093/infdis/160.3. 490

56. Gardner HL, Dukes CD (1955) Haemophilus vaginalis vaginitis: a newly defined specific infection previously classified nonspecific vaginitis. Am J Obstet Gynecol 69(5):962-976

57. Scott TG, Smyth CJ, Keane CT (1987) In vitro adhesiveness and biotype of Gardnerella vaginalis strains in relation to the occurrence of clue cells in vaginal discharges. Genitourin Med 63(1):47-53. https://doi.org/10.1136/sti.63.1.47

58. Gilbert NM, Lewis WG, Lewis AL (2013) Clinical features of bacterial vaginosis in a murine model of vaginal infection with Gardnerella vaginalis. PLoS ONE 8(3):e59539. https://doi.org/ 10.1371/journal.pone.0059539

59. Cauci S, Hitti J, Noonan C, Agnew K, Quadrifoglio F, Hillier SL et al (2002) Vaginal hydrolytic enzymes, immunoglobulin A against Gardnerella vaginalis toxin, and risk of early preterm birth among women in preterm labor with bacterial vaginosis or intermediate flora. Am J Obstet Gynecol 187(4):877-881. https:// doi.org/10.1067/mob.2002.127454

60. Cauci S, Thorsen P, Schendel DE, Bremmelgaard A, Quadrifoglio F, Guaschino S (2003) Determination of immunoglobulin A against Gardnerella vaginalis hemolysin, sialidase, and prolidase activities in vaginal fluid: implications for adverse pregnancy outcomes. J Clin Microbiol 41(1):435-438. https://doi.org/ 10.1128/jcm.41.1.435-438.2003

61. Srinivasan U, Misra D, Marazita ML, Foxman B (2009) Vaginal and oral microbes, host genotype and preterm birth. Med Hypotheses 73(6):963-975. https://doi.org/10.1016/j.mehy.2009. 06.017

62. McGregor JA, French JI, Jones W, Milligan K, McKinney PJ, Patterson E et al (1994) Bacterial vaginosis is associated with prematurity and vaginal fluid mucinase and sialidase: results of a controlled trial of topical clindamycin cream. Am J Obstet Gynecol 170(4):1048-59. https://doi.org/10.1016/s00029378(94)70098-2 (discussion 59-60)

63. Lewis AL, Lewis WG (2012) Host sialoglycans and bacterial sialidases: a mucosal perspective. Cell Microbiol 14(8):11741182. https://doi.org/10.1111/j.1462-5822.2012.01807.x

64. Cauci S, Culhane JF, Di Santolo M, McCollum K (2008) Among pregnant women with bacterial vaginosis, the hydrolytic enzymes sialidase and prolidase are positively associated with interleukin1beta. Am J Obstet Gynecol 198(1):132.e1-7. https://doi.org/10. 1016/j.ajog.2007.05.035

65. Thurman AR, Doncel GF (2011) Innate immunity and inflammatory response to Trichomonas vaginalis and bacterial vaginosis: relationship to HIV acquisition. Am J Reprod Immunol 65(2):89-98. https://doi.org/10.1111/j.1600-0897.2010.00902.x

66. Amith SR, Jayanth P, Franchuk S, Finlay T, Seyrantepe V, Beyaert $R$ et al (2010) Neu1 desialylation of sialyl alpha-2,3-linked beta-galactosyl residues of TOLL-like receptor 4 is essential for receptor activation and cellular signaling. Cell Signal 22(2):314324. https://doi.org/10.1016/j.cellsig.2009.09.038

67. Al-Mushrif S, Eley A, Jones BM (2000) Inhibition of chemotaxis by organic acids from anaerobes may prevent a purulent response in bacterial vaginosis. J Med Microbiol 49(11):1023-1030. https://doi.org/10.1099/0022-1317-49-11-1023

68. Chaudry AN, Travers PJ, Yuenger J, Colletta L, Evans P, Zenilman JM et al (2004) Analysis of vaginal acetic acid in patients undergoing treatment for bacterial vaginosis. J Clin Microbiol 42(11):5170-5175. https://doi.org/10.1128/jcm.42.11.51705175.2004 
69. Castro J, França A, Bradwell KR, Serrano MG, Jefferson KK, Cerca N (2017) Comparative transcriptomic analysis of Gardnerella vaginalis biofilms vs. planktonic cultures using RNAseq. npj Biofilms Microbiomes 3(1):3. https://doi.org/10.1038/ s41522-017-0012-7

70. Janulaitiene M, Paliulyte V, Grinceviciene S, Zakareviciene J, Vladisauskiene A, Marcinkute A et al (2017) Prevalence and distribution of Gardnerella vaginalis subgroups in women with and without bacterial vaginosis. BMC Infect Dis 17(1):394. https:// doi.org/10.1186/s12879-017-2501-y

71. Høiby N, Ciofu O, Johansen HK, Song ZJ, Moser C, Jensen PØ et al (2011) The clinical impact of bacterial biofilms. Int J Oral Sci 3(2):55-65. https://doi.org/10.4248/IJOS11026

72. Machado D, Castro J, Palmeira-de-Oliveira A, Martinez-deOliveira J, Cerca N (2015) Bacterial vaginosis biofilms: challenges to current therapies and emerging solutions. Front Microbiol 6:1528. https://doi.org/10.3389/fmicb.2015.01528

73. Swidsinski A, Mendling W, Loening-Baucke V, Swidsinski S, Dörffel Y, Scholze J et al (2008) An adherent Gardnerella vaginalis biofilm persists on the vaginal epithelium after standard therapy with oral metronidazole. Am J Obstetr Gynecol 198(1):97. https://doi.org/10.1016/j.ajog.2007.06.039

74. Alves P, Castro J, Sousa C, Cereija TB, Cerca N (2014) Gardnerella vaginalis outcompetes 29 other bacterial species isolated from patients with bacterial vaginosis, using in an in vitro biofilm formation model. J Infect Dis 210(4):593-596. https://doi.org/10. 1093/infdis/jiu131

75. Bradshaw CS, Brotman RM (2015) Making inroads into improving treatment of bacterial vaginosis-striving for longterm cure. BMC Infect Dis 15(1):292. https://doi.org/10.1186/ s12879-015-1027-4

76. Swidsinski A, Mendling W, Loening-Baucke V, Ladhoff A, Swidsinski S, Hale LP et al (2005) Adherent biofilms in bacterial vaginosis. Obstet Gynecol 106(5 Pt 1):1013-1023. https:// doi.org/10.1097/01.AOG.0000183594.45524.d2

77. Bradshaw CS, Morton AN, Hocking J, Garland SM, Morris MB, Moss LM et al (2006) High recurrence rates of bacterial vaginosis over the course of 12 months after oral metronidazole therapy and factors associated with recurrence. J Infect Dis 193(11):1478-1486. https://doi.org/10.1086/503780

78. Hymes SR, Randis TM, Sun TY, Ratner AJ (2013) DNase Inhibits Gardnerella vaginalis Biofilms In Vitro and In Vivo. J Infect Dis 207(10):1491-1497. https://doi.org/10.1093/infdis/jit047

79. Machado A, Jefferson KK, Cerca N (2013) Interactions between Lactobacillus crispatus and bacterial vaginosis (BV)-associated bacterial species in initial attachment and biofilm formation. Int J Mol Sci 14(6):12004-12012. https://doi.org/10.3390/ijms140612004

80. Machado A, Almeida C, Salgueiro D, Henriques A, Vaneechoutte M, Haesebrouck F et al (2013) Fluorescence in situ hybridization method using peptide nucleic acid probes for rapid detection of lactobacillus and Gardnerella spp. BMC Microbiol 13:82. https://doi.org/10.1186/1471-2180-13-82

81. Hardy L, Jespers V, Abdellati S, De Baetselier I, Mwambarangwe L, Musengamana $\mathrm{V}$ et al (2016) A fruitful alliance: the synergy between Atopobium vaginae and Gardnerella vaginalis in bacterial vaginosis-associated biofilm. Sex Transm Infect 92(7):487491. https://doi.org/10.1136/sextrans-2015-052475

82. Castro J, Henriques A, Machado A, Henriques M, Jefferson KK, Cerca N (2013) Reciprocal interference between Lactobacillus spp. and Gardnerella vaginalis on initial adherence to epithelial cells. Int J Med Sci. 10(9):1193-8. https://doi.org/10.7150/ijms.6304

83. Machado A, Cerca N (2015) Influence of biofilm formation by gardnerella vaginalis and other anaerobes on bacterial vaginosis. J Infect Dis 212(12):1856-1861. https://doi.org/10.1093/infdis/ jiv338
84. Castro J, Cerca N (2015) BV and non-BV associated Gardnerella vaginalis establish similar synergistic interactions with other BVassociated microorganisms in dual-species biofilms. Anaerobe 36:56-59. https://doi.org/10.1016/j.anaerobe.2015.10.008

85. Pybus V, Onderdonk AB (1997) Evidence for a commensal, symbiotic relationship between Gardnerella vaginalis and Prevotella bivia involving ammonia: potential significance for bacterial vaginosis. J Infect Dis 175(2):406-413. https://doi.org/10.1093/ infdis/175.2.406

86. Castro J, Machado D, Cerca N (2016) Escherichia coli and Enterococcus faecalis are able to incorporate and enhance a preformed Gardnerella vaginalis biofilm. Pathog Dis 74(3):ftw007. https://doi.org/10.1093/femspd/ftw007

87. Patterson JL, Stull-Lane A, Girerd PH, Jefferson KK (2010) Analysis of adherence, biofilm formation and cytotoxicity suggests a greater virulence potential of Gardnerella vaginalis relative to other bacterial-vaginosis-associated anaerobes. Microbiology (Reading) 156(Pt 2):392-399. https://doi.org/10.1099/mic.0. 034280-0

88. Govinden G, Parker JL, Naylor KL, Frey AM, Anumba DOC, Stafford GP (2018) Inhibition of sialidase activity and cellular invasion by the bacterial vaginosis pathogen Gardnerella vaginalis. Arch Microbiol 200(7):1129-1133. https://doi.org/10. 1007/s00203-018-1520-4

89. Castro J, Martins AP, Rodrigues ME, Cerca N (2018) Lactobacillus crispatus represses vaginolysin expression by BV associated Gardnerella vaginalis and reduces cell cytotoxicity. Anaerobe 50:60-63. https://doi.org/10.1016/j.anaerobe.2018.01.014

90. Machado A, Salgueiro D, Harwich M, Jefferson KK, Cerca N (2013) Quantitative analysis of initial adhesion of bacterial vaginosis-associated anaerobes to ME-180 cells. Anaerobe 23:1-4. https://doi.org/10.1016/j.anaerobe.2013.07.007

91. Patterson JL, Girerd PH, Karjane NW, Jefferson KK (2007) Effect of biofilm phenotype on resistance of Gardnerella vaginalis to hydrogen peroxide and lactic acid. Am J Obstet Gynecol 197(2):170-177. https://doi.org/10.1016/j.ajog.2007.02.027

92. Ishida A, Akita K, Mori Y, Tanida S, Toda M, Inoue M et al (2014) Negative regulation of Toll-like receptor-4 signaling through the binding of glycosylphosphatidylinositol-anchored glycoprotein, CD14, with the sialic acid-binding lectin, CD33. J Biol Chem 289(36):25341-25350. https://doi.org/10.1074/jbc. M113.523480

93. Stamatos NM, Carubelli I, van de Vlekkert D, Bonten EJ, Papini N, Feng C et al (2010) LPS-induced cytokine production in human dendritic cells is regulated by sialidase activity. J Leukoc Biol 88(6):1227-1239. https://doi.org/10.1189/jlb.1209776

94. Bornhöfft KF, Goldammer T, Rebl A, Galuska SP (2018) Siglecs: a journey through the evolution of sialic acid-binding immunoglobulin-type lectins. Dev Comp Immunol 86:219-231. https:// doi.org/10.1016/j.dci.2018.05.008

95. Myziuk L, Romanowski B, Johnson SC (2003) BVBlue test for diagnosis of bacterial vaginosis. J Clin Microbiol 41(5):19251928. https://doi.org/10.1128/jcm.41.5.1925-1928.2003

96. Bradshaw CS, Morton AN, Garland SM, Horvath LB, Kuzevska I, Fairley CK (2005) Evaluation of a point-of-care test, BVBlue, and clinical and laboratory criteria for diagnosis of bacterial vaginosis. J Clin Microbiol 43(3):1304-1308. https://doi.org/ 10.1128/jcm.43.3.1304-1308.2005

97. Amabebe E, Reynolds S, Stern V, Stafford G, Paley M, Anumba DOC (2016) Cervicovaginal fluid acetate: a metabolite marker of preterm birth in symptomatic pregnant women. Front Med 3:48. https://doi.org/10.3389/fmed.2016.00048

98. Amabebe E, Reynolds S, Stern VL, Parker JL, Stafford GP, Paley MN et al (2016) Identifying metabolite markers for preterm birth in cervicovaginal fluid by magnetic resonance 
spectroscopy. Metabolomics 12(4):67. https://doi.org/10.1007/ s11306-016-0985-x

99. Amabebe E, Reynolds S, He X, Wood R, Stern V, Anumba DOC (2019) Infection/inflammation-associated preterm delivery within 14 days of presentation with symptoms of preterm labour: a multivariate predictive model. PLoS ONE 14(9):e0222455. https://doi.org/10.1371/journal.pone.0222455

100. Galleh RP, Chen X, Lambert DW, Stafford GP (2020) Testing for novel inhibitors of periodontitis-associated sialidases. Access Microbiol. https://doi.org/10.1099/acmi.ac2020.po0070

Publisher's Note Springer Nature remains neutral with regard to jurisdictional claims in published maps and institutional affiliations. 